\title{
V Conferencia Colombiana de Control Automático de IEEE: Un punto de encuentro entre la industria, la academia y la investigación
}

\section{Fifth Colombian Conference on IEEE Automatic Control: A meeting point for industry, academy and research}

\section{Valentina Cárdenas Cadena*}

O

Recepción: 10/11/2021
.

Aprobación: 26/11/2021
O

Publicación: 22/12/2021

\section{Para citar este artículo}

Cárdenas Cadena, V. (2021). V Conferencia Colombiana de Control Automático de IEEE: Un punto de encuentro entre la industria, la academia y la investigación. Indagare, (9), 109-114. https://doi.org/10.35707/indagare/912

\footnotetext{
1 * Programa de Comunicación Social y Periodismo de la Universidad de Ibagué, Ibagué, Colombia.
} ORCID: 0000-0001-8569-6745. Correo electrónico: cardenas.valentina0@gmail.com 


\title{
Resumen
}

En el marco de las distintas actividades que desarrolla el Instituto de Ingenieros Eléctricos y Electrónicos (IEEE), en octubre se celebró la v Conferencia Colombiana de Control Automático, organizada por la Universidad de Ibagué y cinco universidades más. Esta conferencia llena de retos para los organizadores, tras de ser la primera en realizarse de manera virtual, también fue la oportunidad perfecta para que todos los interesados en el control y la automatización industrial conocieran y estuvieran al tanto de las últimas novedades en esta área temática.

\begin{abstract}
As part of the various activities developed by the Institute of Electrical and Electronics Engineers (IEEE), in October the Fifth Colombian Conference on Automatic Control was held and organized by Universidad de Ibagué and other five universities. This conference was full of challenges for the organizers, after being the first to be held virtually, it was also the perfect opportunity for all those interested in industrial control and automation knew and were aware of the latest developments in this subject area.
\end{abstract}

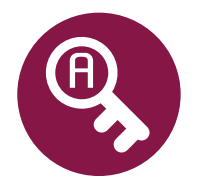

Palabras claves: Electrónica, control, conferencia, investigación, industria.

Key words: Electronics, control, conference, research, industry.

Del 19 al 22 de octubre de 2021, se llevó a cabo la V Conferencia Colombiana de Control Automático, un evento bianual que reúne docentes, estudiantes, investigadores e industriales interesados en los avances y tendencias de la ingeniería de control. Esta conferencia fue un punto de encuentro de esta comunidad, en la que se compartieron experiencias relacionadas con la aplicación y teoría del control automático en la academia, la investigación y la industria.

Aunque la conferencia se realizó de manera virtual, Ibagué fue la ciudad designada como plaza con la participación del docente Oswaldo López Santos del programa de Ingeniería Electrónica de la Universidad de Ibagué, quien tuvo el rol de codirector general (co-chair).

La revista Indagare tuvo la oportunidad de hablar con el profesor Oswaldo, quien compartió con nosotros los retos y los aprendizajes de esta experiencia sin precedentes. 
Figura 1. Retrato de Oswaldo López Santos

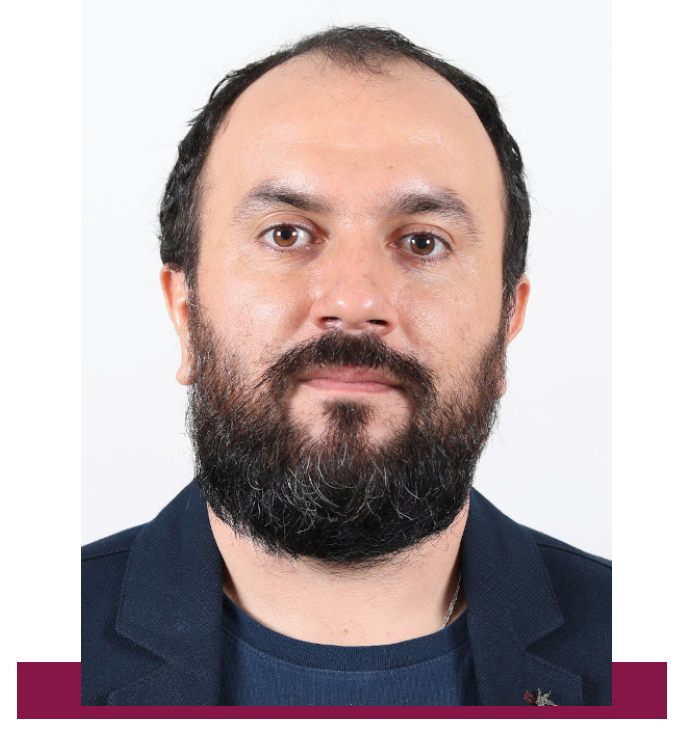

Fuente: Comunicación institucional, Universidad de Ibagué

\section{¿Cómo logró el puesto de codirector general (co-chair)?}

En principio, había que ofrecer la plaza de la ciudad para hacer la conferencia, así que propuse la ciudad de Ibagué. Seguido a esto, hay una comunidad, el Capítulo Colombiano de la Sociedad de Sistemas de Control Automático del IEEE, que te da el voto de confianza para coordinar la conferencia, claro está con la participación activa de otras personas y universidades.

La selección consiste en que, primero, te arriesgues a hacerlo, y segundo, en que tengas una visibilidad y una trayectoria que sea evidencia de tus capacidades y tu responsabilidad para liderar esta conferencia. Sin embargo, no estás solo en esta organización. Nosotros éramos un equipo de trabajo plano: entre todos hacemos todo. Los roles sí tienen una jerarquía: el rol de responsabilidad más alto lo compartía yo con la profesora Diana Marcela Ovalle de la Universidad Distrital Francisco José de Caldas, por eso el prefijo "co" en "codirector" (co-chair) porque éramos dos directores generales, y hacía abajo estaban otros responsables de cuestiones particulares dentro de la conferencia.

\section{¿Qué es el Instituto de Ingenieros Eléctricos y Electrónicos (IEEE)?}

Es una asociación internacional, hoy en día la más importante para ingeniería eléctrica y electrónica. En ella hacen parte más 420000 docentes, estudiantes, investigadores dedicados a la innovación y avance de temas científico-técnicos. 
El IEEE cuenta con una editorial, en la que se editan revistas muy importantes para las diferentes áreas de ingeniería eléctrica y electrónica, entre ellas, por supuesto, el control automático.

La Conferencia Colombiana de Control Automático hace parte de las actividades que desarrolla el IEEE en Colombia.

\section{¿Qué actividades se realizaron en la v Conferencia Colombiana de Control Automático?}

Se realizaron cinco tipos de actividades académicas. En primer lugar, las charlas centrales dictadas por conferencistas especializados. Se contó con la participación de ocho conferencistas internacionales (Estados Unidos, Bélgica, Francia y Dinamarca) y dos nacionales, todos ellos aportaron charlas de muy alta calidad. La segunda actividad, fueron los talleres. Estos son un poco más extensos en tiempo y su objetivo era generar más interacción con el público. La tercera actividad académica fueron las sesiones paralelas, en las cuales se exponían los artículos de investigación relacionados al área de control automático.

Para esta edición, tuvimos una actividad especial llamada "controlavirus challenge" direccionada a estudiantes que pretendían conocer propuestas en el área de control para el manejo de la CoviD-19. También tuvimos una sesión de pósteres dirigida a estudiantes de doctorado.

Adicional a estas actividades académicas, la conferencia contaba con una ceremonia de apertura, cierre y una actividad cultural, en la cual se contó con la participación de los ensambles musicales de la Universidad de Ibagué, escenario que los asistentes disfrutaron mucho.

\section{¿Cómo fue la participación de autores en esta conferencia?}

Tuvimos una buena participación para estar en medio de una pandemia y tener una conferencia virtual. Normalmente, uno de los atractivos de asistir a estos eventos es precisamente viajar al lugar de las conferencias, lo que en esta ocasión nos jugaba en contra. Por eso, pensamos que nos fue muy bien, recibimos 72 trabajos de investigación. Usualmente se presentan cerca de 100, pero en las circunstancias que teníamos, recibir este número de trabajos, no lo vemos como si nos hubiesen faltado, sino como: "no es lo mismo que ha pasado antes, pero para las condiciones que se dieron, estuvo bien".

Desde la Universidad de Ibagué también participaron algunos docentes, quienes aportaron los diferentes avances de sus proyectos en varias áreas. Yo trabajo en el área de control, pero aplicado a energía. Hay profesores que trabajan en control, enfocados en robótica, percepción e instrumentación. A partir de los resultados que arrojan los proyectos, se plantea y se somete un artículo para que lo evalúen unos pares, 
que son otros profesores e investigadores, quienes deciden aceptar o no el artículo para presentarse a la conferencia. De esta forma se reciben comentarios y críticas que permiten que mejore la calidad de tu proyecto.

\section{¿Deberían los estudiantes participar de esta conferencia?}

En los artículos normalmente siempre hay un estudiante, y en muchos de los casos, quien presenta el artículo en la conferencia, es el estudiante. Eso quiere decir que gran parte de la gente que asiste son estudiantes de pregrado o posgrado. Es una actividad en la que ellos tienen un rol muy importante.

Las conferencias de este nivel son muy enriquecedoras para cualquier participante. Todo lo que uno puede apreciar es muy valioso y complementa lo que uno va haciendo en la Universidad. Es muy importante que los estudiantes participen como asistentes en este tipo de actividades, que se animen a enterarse sobre qué está pasando allí. También, que aporten sus diferentes trabajos de investigación y produzcan artículos que puedan llevarse a la conferencia y, así mismo, ser una fuente de participación de estudiantes y profesores.

\section{¿Cómo se compara esta $\mathrm{v}$ edición de la conferencia con las anteriores?}

La experiencia de estar en el lugar es diferente. Es más fácil establecer contacto e interactuar con los otros. Pero las demás actividades se desarrollan de la misma manera: asistir a las salas, conferencias, hacer preguntas, participar de talleres. En la virtualidad se podía hacer algo similar.

Las conferencias incluyen una actividad cultural en la que se comparte una cena. $Y$ es en ese momento en el que la gente come u observa un grupo de danza, que se generan conversaciones interesantes u oportunidades de trabajo conjunto. Lo anterior es más difícil en lo virtual, aunque lo intentamos por medio de unas salas de conversaciones virtuales, en las conferencias presenciales es más fácil. Sin embargo, la virtualidad pudo facilitar un mayor contacto con los conferencistas internacionales. Nosotros contamos con la participación de diez conferencistas. En una conferencia presencial normalmente asisten tres o máximo cuatro. Es difícil tener reunidos en una misma conferencia las personas de la talla y calidad que trajimos, así que, por ese lado, la virtualidad tiene una ventaja efectiva.

\section{¿Cómo fue para usted esta experiencia?}

Fue un aprendizaje total. Yo no había participado en la organización de otra conferencia, por ello es un aprendizaje completo. La experiencia consiste en ir dándose cuenta de todos los pequeños detalles que tienen que afinarse para que el evento salga bien, de cómo integrar las ideas que tienen las demás personas del equipo, de observar 
cómo fluyen y garantizar que así sigan, y ser consciente de cómo las cosas se van organizando. También es aprender de las limitaciones que se tienen, a veces quieres hacer un montón de cosas, pero en realidad no se cuenta con los recursos necesarios, entre ellos el tiempo.

En términos generales, es un aprendizaje muy interesante, y una alegría cuando se logra. Tú vas enfrentando todas las pequeñas cosas, y en el momento en el que llega la conferencia, no hay vuelta atrás. Esta fluye, y es bastante satisfactorio que todo salga tal como se tenía pensado y preparado desde hace más de un año.

La invitación final para la comunidad interesada es participar de espacios como la Conferencia Colombiana de Control Automático, ser parte de la IEEE y de sus actividades. Estas son oportunidades para profesionales, investigadores, docentes, y estudiantes para aprender y potencializar sus intereses, pero sobre todo para darse a conocer y generar proyectos en conjunto. El interés de estar en sociedad es el de trabajar con otros y construir de manera colectiva. 